\title{
IMPORTANCE OF THE HIGH ENERGY CHANNEL FOR THE GAMMA-RAY BURST DATA
}

\author{
A. MÉSZÁROS \\ Dpt. Astron., Charles Univ., Prague 5, Švédská 8, Czech Rep. \\ and Konkoly Obs., Budapest, Box 67, H-1525, Hungary \\ Z. BAGOLY \\ Eötvös Univ., Lab. Inform. Technol., Múzeum krt.6-8, H-1088 \\ Budapest, Hungary \\ L. G. BALÁZS \\ Konkoly Obs., Budapest, Box 67, H-1525, Hungary \\ AND \\ I. HORVÁTH, P. MÉSZÁROS \\ Dpt. Astron., Penn. State Univ., 525 Davey Lab., University \\ Park, PA 16802, USA
}

\section{Principal Component Analysis}

Extensive data bases on Gamma-Ray Burst (GRB) properties such as the BATSE 3B catalog (Meegan et al. 1996) contain a wealth of statistical information. The nine entries of the 3B database for each GRB consist of two durations, $T_{50}, T_{90}$, which contain $50 \%$ and $90 \%$ of the burst energy, respectively; four fluences (time-integrated energy fluxes) $F 1, F 2, F 3, F 4$, defined over different energy channels; and three measures of the peak flux (each summed over the four energy channels), measured over three different resolution timescales $(64 \mathrm{~ms}, 256 \mathrm{~ms}$ and $1024 \mathrm{~ms})$. Thus the initial number of variables is $n=9$. There is, of course, some incompleteness in the catalog. There are 625 GRBs having all 9 non-zero quantities, and only they are considered here.

The Principal Component Analysis (PCA) is done on these. This statistical procedure is explained in (Jolliffe 1986) and (Kendall \& Stuart 1976). It can determine the number $m$ of important quantities $(m<n)$. 


\section{Results}

The results of this procedure are the following. The first PC is roughly given by the sum of all the quantities, with some extra weight on the first three fluences. Because of the different dimensions involved, it has only a formal meaning. The second PC, accounting for $26.5 \%$ of the variation, is clearly important, this value being far above Jolliffe's $70 / n=7.8 \%$ cut-off level (Jolliffe 1972). This PC is roughly given by the formal difference of the logarithmic peak fluxes and durations. Hence, the duration, peak flux and total fluence are undoubtedly important quantities, but only two of them are independent. This means that in the roughest approximation, it is enough to consider, e.g., a total fluence and a duration, and these two represent $91.6 \%$ of the information content in the $3 \mathrm{~B}$ catalog. The third PC, practically defined by $F 4$ alone, accounts for $5.1 \%$ of the variation and is already below Jolliffe's level. Hence $m=2$. Because it is just below Jolliffe's level, this third PC might however be of some importance. The fourth PC, with its $1.5 \%$, is far below Jolliffe's limit, and is unimportant.

Some of the results are unexpected. For instance, the analysis indicates that an allowable approximation would be to combine (add) the fluences in the first three channels, and consider them in conjunction with the fluence F4 in the fourth channel as the basis vectors for the fluence space. This singling out of $F 4$ based on its information content appears to be new.

The details of this contribution will be presented elsewhere (Bagoly et al. 1997).

This research was supported by Domus Hungarica Scientiarium Artiumque Foundation and Charles University grant 101-10/736 (A.M.), NASA grants NAG5-2362, NAG5-2857 (P.M., I.H.), OTKA grants F14324, T14304, F26666 (I.H.), T024027 (L.G.B.), KOSEF grant and Széchenyi Foundation (I.H.). I.H. and P.M. are grateful to E.D. Feigelson and E.E. Fenimore for discussions, and A.M. acknowledges the kind hospitality of Konkoly Observatory.

\section{References}

Bagoly, Z., et al. (1997) A Principal Component Analysis of the 3B Gamma-Ray Burst Data, Astrophys. J., submitted for publication.

Jolliffe, I. T. (1972) Discarding Variables in a Principal Component Analysis, I: Artificial data, Appl. Statist., 21, 160-173.

Jolliffe, I. T. (1986) Principal Component Analysis. Springer, New York.

Kendall, M. and Stuart, A. (1976) The Advanced Theory of Statistics. Griffin, London. Meegan, C. A., et al. (1996) The Third BATSE 3B Gamma-Ray Catalog, Astrophys. J. Suppl., 106, 65-110. 


\section{Session 4: Large Scale Hot Plasmas and Their Relation with Dark Matter}

